\title{
Effect of Evidence-Based Method Clinical Education on Patients Care Quality and Their Satisfaction
}

\author{
Morteza Alibakhshi Kenari \\ Martyr Beheshti University of Medical Sciences and Health Services, Tehran, Iran \\ Email: Morteza.alibakhshikenari@gmail.com
}

Received December 26 $6^{\text {th }}, 2013$; revised January 26 $6^{\text {th }}, 2014$; accepted February $4^{\text {th }}, 2014$

\begin{abstract}
Copyright (C) 2014 Morteza Alibakhshi Kenari. This is an open access article distributed under the Creative Commons Attribution License, which permits unrestricted use, distribution, and reproduction in any medium, provided the original work is properly cited. In accordance of the Creative Commons Attribution License all Copyrights (C) 2014 are reserved for SCIRP and the owner of the intellectual property Morteza Alibakhshi Kenari. All Copyright $@ 2014$ are guarded by law and by SCIRP as a guardian.
\end{abstract}

\begin{abstract}
Nowadays, evidence-based education with a serious purpose, more explicit and rational than the best current evidence to decision-making in nursing education, has been addressed. This study aimed to assess the effect of clinical evidence based on the fact that the quality of patient care has been performed and the usual care based on traditional evidence-based care training has been under almost identical. Student feedback questionnaire data, patient satisfaction and quality of care were collected and then were analyzed with descriptive and inferential statistics. This study suggests that using evidence-based education in nursing care is effective not only as traditional education, but also as knowledge and skills, and the quality of patient care increases. On the other hand, it will reduce costs and length of stay in the hospital.
\end{abstract}

Keywords: Evidence-Based Nursing; Student Nurse; Patient Care; Patient Satisfaction

\section{Introduction}

Nurses make up the largest provider of health services and essential role in maintaining and improving health care and health care delivery systems at different levels of play (AdibHagbaghery et al., 2004). Continuing advances in scientific knowledge and technical expertise combined with their professional and scientific evidence and the practice in multidisciplinary groups, clients identify problems, and design, implement and evaluate programs of care for these problems to be solved (Salsali, 2004). Nurses whose actions were based on scientific evidence, have been able to make better decisions, higher quality care to shorten patient's hospital stay and reduce costs, and bring better cost effectiveness for the patient (McKenna, 2004).

Sackett and colleagues' (2000) application of evidence-based care with research knowledge, expertise, clinical experience and patient values in providing care to patients has been known (Sackett \& Rosenberg, 2000). Gibbs (2007) Priorities for evidence-based nursing, placing the interests of patients through the use of best evidence for clinical decisions in patient care, have been defined (Gibbs, 2007). Care based on evidence-based nursing care is progressive, but due to lack of skills in all cases, this approach is not used. The use of evidence-based education on nursing students of high performance and good results (Oh et al., 2010) in one study, increases in the prevention of venous thrombosis patients (Duff et al., 2010). In another study of women with early breast cancer, chemotherapy, reduction and increasing patient self-doubt and uncertainty have been reported. In order to prepare for employment, nursing students need skills to evaluate and use research findings to gain (Balakas \& Sparks, 2010). The evidence-based methods of pain relief were accomplished through the work by Carlson (2010). The results of this study show that users use evidence-based education to increase students' academic scores (Brown et al., 2010). According to the order, $\mathrm{Li}$ et al. use evidence to solve problems that are faced by workers in the real environment ( $\mathrm{Li}$ et al., 2009). Forrest also learned the components of evidence-based, in terms of needs, problems, clinical questions and search strategies in response to the health problems of patients (Smith et al., 2000). These methods are effective ways to meet the needs and problems of care and improve clinical decisions (Melnyk \& Overholt, 2005). Ghanizadeh and colleagues also showed that the ability to update data in an inclusive sense of self is to address clinical issues (Green, 2000). Green indicates the findings of clinical scenarios and discussions led by students, the effectiveness of training (Adib-Hajbaghery et al., 2010). Abedini et al. stated in their study that the use of evidence in clinical practice linking theoretical and empirical concepts and according to the theoretical literature as well as the progress of scientific knowledge is not possible to simply practice and learn fitness professional involved in the real environment (Abedini et al., 2012). Considine \& McGillivray study employed evidencebased care for stroke patients to improve care and better health outcomes (Considine \& McGillivray, 2010). Carlson study showed that the use of evidence-based care for reducing anxiety in cancer patients beginning chemotherapy is highly effective (Carlson, 2010). This study revealed that Drew and his instructions are used to prepare the best available evidence, and that nursing care can lead patients correctly (Drew et al., 2004). McKinley et al. also reduce the use of research evidence that has crashed in the hospital (McKinley et al., 2007). 


\section{Methods}

In this study, the population in 2013 was an undergraduate nursing students, 40 of Seven semesters nursing undergraduate student for the internship Imam Hossein hospital in Tehran admitted to the ward, Before entering the hospital with purposive sampling, based on the average overall grade point average, scores past academic semester internships and gender, were randomly divided into 4 groups of 10.2 groups based on the traditional and usual care group 2 according to the same evidence-based care intervention were instructed by a faculty mentor and each group spent a week training. Data were collected by two questionnaires and a check list. Check list "Assessing the quality of patient care”, which was completed by a clinical instructor in the department, comments questionnaire of students' Factors affecting clinical care "was completed by the students and "Patient satisfaction with clinical care, education and communication" by the patient or by the illiterate and he was perfect.

Check List three areas of clinical care, practical skills using the theory of care and patient education are included this check list has not been informed by the instructor that the main objectives of the study were completed during the care sector. Grading check lists of 10 - 100 (10 - 40 "inappropriate care”, 41 - 70 "Medium Care" and 71 - 100 "optimal care”), respectively. Questionnaire to assess the students' opinion about the factors affecting clinical care, includes 20 questions related to decision making and clinical practice based on the needs of patients, and considering all the patients and the general attitude of the patient's anxiety during the performance, predict a patient's condition, creativity in performance, benefits and drawbacks of each method on the basis of performance and are evaluating the options, pros and cons. Percentage of total responses for each question on the pros and cons of each group were presented in the results. Cronbach reliability coefficient of $86 \%$ was achieved in the preliminary study.

Comments and patient satisfaction questionnaire included 28 questions about the service aspects of clinical care, patient satisfaction, communication, education, and options to measure the question low, medium, is high. Each of the options low score 1 , score 2 and 3 are assigned a high score. Grading Scale based on 28 scores "low satisfaction”, 29 - 56 "happy medium" and 57 - 84 "high satisfaction” is. Cronbach’s alpha reliability coefficient was $89 \%$.

The research was conducted to that students learn about various aspects of care at the beginning of each section was determined by questionnaire and then into 2 groups based on the evidence, the 5 sessions of 2 hours, using evidence-based care were trained by the researcher in a clinical trial of the procedure was performed. Also, the traditional teaching on the part of the patient to engage students and they were trained to do routine care. For all groups performed in clinical care, check list was completed by a clinical instructor alike. At the time of patient discharge, opinions and satisfaction questionnaire was completed and at the end of the course the student feedback questionnaire about factors affecting clinical care completed. To participate in this study, all students were given their written consent. After collecting the data have used of SPSS software to confirmation of data normality by the Kolmogorov-Smirnov $(p>0.05)$ with chi-square, T-independent and T-test analysis. Students' knowledge of evidence-based care for patients in the traditional group, at the beginning of the section was not significantly different when compared with each other $(p=0.7)$. The scores of the students in evidence-based patient care $0.6 \pm$ 0.80 and the traditional $5.4 \pm 67.73$ are summarized in Table 1 .

In both study groups after completion of the study, significantly increased awareness of patient care .Out knowledge of the group based on the evidence before and after the study were $15.47 \pm 58.45$ and $10.91 \pm 71.8$ and the traditional $59.05 \pm 15.8$ and $67.8 \pm 13.89(p=0.01)$.

Two groups of factors influencing students' opinions about patient care are summarized in Table 2.

The evidence-based group, $70 \%$ of patients in the clinical care, patient education, and $80 \%$ after $75 \%$ in relation to the patient's satisfaction was high. Traditional high level of satisfaction in patients in the clinical care of $65 \%, 60 \%$, and in the education of the patient after patient contact was $75 \%$. In the case of education, the significant difference observed between the two groups based on traditional evidence was $(p=0.01)$.

\section{Results}

Evidence-Based Nursing is considered as one of the modern methods of instruction. This study aims to determine the impact of evidence-based nursing skills, nursing process took place that indicates the use of evidence-based nursing due to new scientific evidence, Outdated information and method and time to improve and update the necessary measures. Therefore, it is important to learn the technique and its use in the clinical setting. Because nursing students and nurses each day reliable information on diagnosis, treatment, prognosis, prevention, investigation and identification, planning, implementation and evaluation needs. The traditional information sources are inadequate and often outdated. This approach leads to improved critical thinking, independent clinical decisions and ultimately improve the quality of nursing care. Thus optimizing the use of research in nursing with emphasis on evidence-based nursing leads to the acquisition of professional identity and the quality of nursing care. Another objective of this study was to demonstrate the clinical training of evidence based on the quality of patient care and satisfaction. The results showed that two groups of students based on traditional evidence of practical skills in providing care to patients who are not significantly different from each other. But the dimensions of patient education and the use of theory to practice evidence-based clinical care differences are statistically significant. Among other results of this study to improve student learning by integrating evidence-based care programs are taught. Students in this study believe that evidence-based approach with greater creativity, higher awareness and better diagnosis of care is associated with patient care needs.

Among the major obstacles in using evidence-based approach in this study can be cited: Evidence is difficult to obtain due to limited access to the internet and publications, a number of

Table 1.

Compared to the mean scores of different groups within traditional evidence based care.

\begin{tabular}{ccccc}
\hline Group care field & $\begin{array}{c}\text { Based on } \\
\text { evidence }\end{array}$ & Traditional & $\mathrm{T}$ & $\begin{array}{c}\text { Significance } \\
\text { level }\end{array}$ \\
\hline Practical skills & $73 \pm 5.83$ & $75 \pm 5.83$ & -1.2 & 0.23 \\
Using the theoretical & $82 \pm 5.29$ & $70 \pm 4.25$ & 7.6 & 0.01 \\
Patient education & $85 \pm 6.88$ & $76 \pm 4.26$ & 5.8 & 0.04 \\
\hline
\end{tabular}


Table 2.

Evidence-based and traditional students opinions about factors affecting patient care (numbers outside parentheses are the number and percentage in parentheses.

\begin{tabular}{|c|c|c|c|c|c|}
\hline \multirow{2}{*}{ Group Benefits Index } & \multicolumn{2}{|c|}{ Based on evidence } & \multicolumn{2}{|c|}{ Traditional } & \multirow{2}{*}{ Significance leve } \\
\hline & Against & Compliant & Against & Compliant & \\
\hline Decisions based on patient needs and clinical practice & (30) 6 & (70) 14 & (55) 11 & (45) 9 & 0.05 \\
\hline Considering all the problems in patient care & (25) 5 & (75) 15 & (60) 12 & (40) 8 & 0.02 \\
\hline Reduce anxiety during treatment & (40) 8 & (60) 12 & (55) 11 & (45) 9 & 0/02 \\
\hline Status in predicting patient care & (40) 8 & (60) 12 & (70) 14 & (30) 6 & 0.01 \\
\hline Full discretion of the patient & (20) 4 & (80) 16 & (50) 10 & (50) 10 & 0.01 \\
\hline Creativity in Care & (30) 6 & (70) 14 & (65) 13 & (65) 13 & 0.01 \\
\hline
\end{tabular}

patients, lack of confidence to make changes, lack of knowledge of nurses, Restrictions on access to research articles and an attitude that was not enough for the care and need to learn new skills and find a lot of resources and detailed evidence. Also, the poll results indicate four major obstacles in the path of nursing care based on the evidence include: The lack of practical nursing research, nurses lack the skills to access and evaluate research evidence, lack of institutional support and time.

\section{Conclusion}

Integration of research evidence in nursing care not only is effective as the traditional approach, it also promotes professsional nursing knowledge, skills and nursing care of high quality and effectiveness. They also reduce the duration and cost of hospitalization. Evidence-based nursing education can strengthen the critical thinking and Developing Creativity over traditional nursing process to promoting skills in nursing students. Therefore, this method can be used in clinical education.

\section{Acknowledgements}

The author would like to express his sincere thanks to Martyr Beheshti University of Medical Sciences and Health Services and Imam Hossein hospital, Tehran, Iran.

\section{REFERENCES}

Abedini, Z., Ahmari, T. H., Khorami, R. A., \& Heidar Pour, A. (2012). Evidence-based nursing students' experiences of learning in the clinical setting. Iranian Journal of Medical Education, 8, 864-872.

Adib-Hagbaghery, M., \& Salsali, M., \& Ahmadi, F. (2004a). A qualitative study of Iranian nurses' understanding and experiences of professional power. Human Resources for Health, 2, 9.

Adib-Hagbaghery, M., \& Salsali, M., \& Ahmadi, F. (2004b). Clinical decision: Way to strengthen the nursing profession. Iranian Journal of Medical Education, 10, 3-12.

Adib-Hagbaghery, M., Salsali, M., \& Ahmadi, F. (2004c). The factors facilitating and inhibiting effective clinical decision-making in nursing: a qualitative study. BMC Nursing, 3, 2. http://dx.doi.org/10.1186/1472-6955-3-2

Adib-Hajbaghery, M. (2007). Factors facilitating and inhibiting evidence-based nursing in Iran. Journal of Advanced Nursing, 58, 566575.

Adib-Hajbaghery, M. (2010). Factors influencing evidence-based nursing: A qualitative study. Iran Journal of Nursing, 19, 3-19.

Balakas, K., \& Sparks, L. (2010). Teaching research and evidencebased practice using a service-learning approach. Journal of Nursing
Education, 49, 691-695.

http://dx.doi.org/10.3928/01484834-20100831-07

Brown, C. E., Kim, S. C., Stichler, J. F., \& Fields, W. (2010). Predictors of knowledge, attitudes, use and future use of evidence-based practice among baccalaureate nursing students at two universities. Nurse Education Today, 30, 521-527.

Carlson, C. L. (2010). Prior conditions influencing nurses' decisions to adopt evidence-based postoperative pain assessment practices. Pain Management Nursing, 11, 245-258. http://dx.doi.org/10.1016/j.pmn.2009.05.003

Considine, J., \& McGillivray, B. (2010). An evidence-based practice approach to improving nursing care of acute stroke in an Australian emergency department. Journal of Clinical Nursing, 19, 138-144. http://dx.doi.org/10.1111/j.1365-2702.2009.02970.x

Drew, B. J., Califf, R. M., Funk, M., Kaufman, E. S., Krucoff, M. W., Laks, M. M., et al. (2004). Practice standards for electrocardiographic monitoring in hospital settings: An American heart association scientific statement from the councils on cardiovascular nursing, clinical cardiology, and cardiovascular disease in the young: Endorsed by the international society of computerized electrocardiology and the American association of critical-care nurses. Circulation, 110, 2721-2746. http://dx.doi.org/10.1161/01.CIR.0000145144.56673.59

Duff, J., Walker, K., \& Omari A. (2010). Translating Venous Thromboembolism (VTE) prevention evidence into practice: A multidisciplinary evidence implementation project. Worldviews on EvidenceBased Nursing, 8, 30-39. http://dx.doi.org/10.1111/j.1741-6787.2010.00209.x

Gibbs, L. (2007). Applying research to making life-affecting judgments and decisions. Research on Social Work Practice, 17, 143-150. http://dx.doi.org/10.1177/1049731506294802

Green, M. L. (2000). Evidence based medicine training in internal medicine residency programs. Journal of General Internal Medicine, 15, 129-133. http://dx.doi.org/10.1046/j.1525-1497.2000.03119.x

Li, Y., Wu, T., Shang, H., \& Yang, K. (2009). Strategies for promoting the development of evidence based medicine in China. Journal of Evidence-Based Medicine, 2, 47-52. http://dx.doi.org/10.1111/j.1756-5391.2009.01012.x

McKenna, H. P., Ashton, S., \& Keeney, S. (2004). Barriers to evidencebased practice in primary care. Journal of Advanced Nursing, 45, 178-189. http://dx.doi.org/10.1046/j.1365-2648.2003.02879.x

McKinley, C., Fletcher A., Biggins A., et al. (2007). Evidence-based management practice: Reducing falls in hospital. Collegian, 14, 2025. http://dx.doi.org/10.1016/S1322-7696(08)60551-X

Melnyk, B. M., \& Fineout-Overholt, E. (2005). Evidence-based practice in nursing \& healthcare (pp. 6-10). Philadelphia, PA: Lippincott Williams \& Wilkins.

Oh, E. G., Kim, S., Kim, S.S., Kim, S., Cho, E. Y., Yoo, J.S., et al. (2010). Integrating evidence-based practice into RN-to-BSN clinical nursing education. Journal of Nursing Education, 49, 387-392. http://dx.doi.org/10.3928/01484834-20100331-02

Sckatt, D., \& Rosenberg, W. (2000). Evidence-based medicine. http://cebm.jrz.ox.ac.uk 
Smith, C. A., Ganschow, P. S., Reilly, B. M., Evans, A. T., McNutt, R. A., Osei, A., et al. (2000). Teaching residents evidence-based medicine skills: A controlled trial of effectiveness and assessment of du- rability. Journal of General Internal Medicine, 15, 710-715. http://dx.doi.org/10.1046/j.1525-1497.2000.91026.x 